\title{
Spinal drop metastasis from a benign fourth ventricular choroid plexus papilloma in a pediatric patient: case report
}

\author{
*Ramin A. Morshed, MD,, Darryl Lau, MD, ${ }^{1}$ Peter P. Sun, MD, ${ }^{2}$ and Lauren R. Ostling, MD² \\ 'Department of Neurological Surgery, University of California, San Francisco; and 2UCSF Benioff Children's Hospital \\ Oakland, California
}

\begin{abstract}
Choroid plexus papillomas (CPPs) are typically benign tumors that can occur in any age group but are more commonly found in pediatric patients. Although these tumors are benign, there are several reports in adult patients of distant metastases present either at the time of diagnosis or occurring months to years after initial resection. Here, the authors report the case of a 14-year-old boy who presented with symptoms of elevated intracranial pressure due to obstructive hydrocephalus that was caused by a large fourth ventricular mass. Preoperative imaging included a full MRI of the spine, which revealed an intradural lesion that encased the distal sacral nerve roots at the tip of the thecal sac and was concerning for a drop metastasis. The patient underwent gross-total resection of both the fourth ventricular and sacral tumors with histology of both lesions consistent with benign CPP (WHO Grade I). In addition, the authors review prior reports of both pediatric and adult patients in whom benign CPPs have metastasized with either benign or atypical pathology found at a distant site. Taking into account this unusual case and reports in the literature, patients with even benign CPPs may warrant initial and routine follow-up imaging of the total neural axis in search of the rare, but possible, occurrence of drop metastasis.
\end{abstract}

https://thejns.org/doi/abs/10.3171/2017.5.PEDS17130

KEY WORDS choroid plexus papilloma; metastasis; disseminated; pediatric; oncology

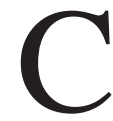
HOROID plexus papillomas (CPPs) are typically extraaxial benign tumors that originate from the neuroepithelial lining of the choroid plexus. These tumors are quite rare, accounting for about $0.4 \%-0.6 \%$ of all intracranial tumors. ${ }^{21,37} \mathrm{CPPs}$ can be found in any age group but are more commonly found in pediatric patients, with a median age at diagnosis of 3.5 years and an incidence of $3.9 \%$ in children younger than 12 years..$^{21,37}$ As the origin of CPP is from the choroid plexus, it can be found anywhere the choroid plexus is located. While an infratentorial location is typical in adults (e.g., fourth ventricle), these tumors are more frequently found in a supratentorial location (e.g., lateral ventricles) in children..$^{15,37}$ Benign CPPs (WHO Grade I) resemble normal choroid plexus with papillary projections containing cuboidal-tocolumnar epithelium lining fibrovascular bundles. While occasional mitoses are present, in general these tumors do not demonstrate cytological atypia. Atypical CPPs (WHO Grade II) demonstrate increased mitotic activity with at least 2 mitoses per $10 \mathrm{hpf}$. Other features may include hypercellularity, cytological atypia, and necrosis. ${ }^{29}$ Given that
CPPs are generally benign and locally slow growing, these tumors are typically cured through gross-total resection alone. 28,30

Although rare, there have been several reported cases of benign CPPs forming distant metastases in adults..$^{1-6}$, 8-10,12-14,16-19,22-27,31-36,38,39 In contrast, there are even fewer reported cases that demonstrate similar findings in the pediatric population. In this report, we present the rare case of a pediatric patient who was found to have a benign fourth ventricular CPP and a sacral drop metastasis at initial presentation. In addition, we review the literature comprehensively and summarize prior reports in both pediatric and adult patients in whom benign CPPs have metastasized to the distant sites.

\section{Case Report}

History and Examination

A 14-year-old boy presented to the emergency department with new complaints of pressure-like headaches and a 2-month history of morning vomiting. On examination,

* Drs. Morshed and Lau contributed equally to this work. 

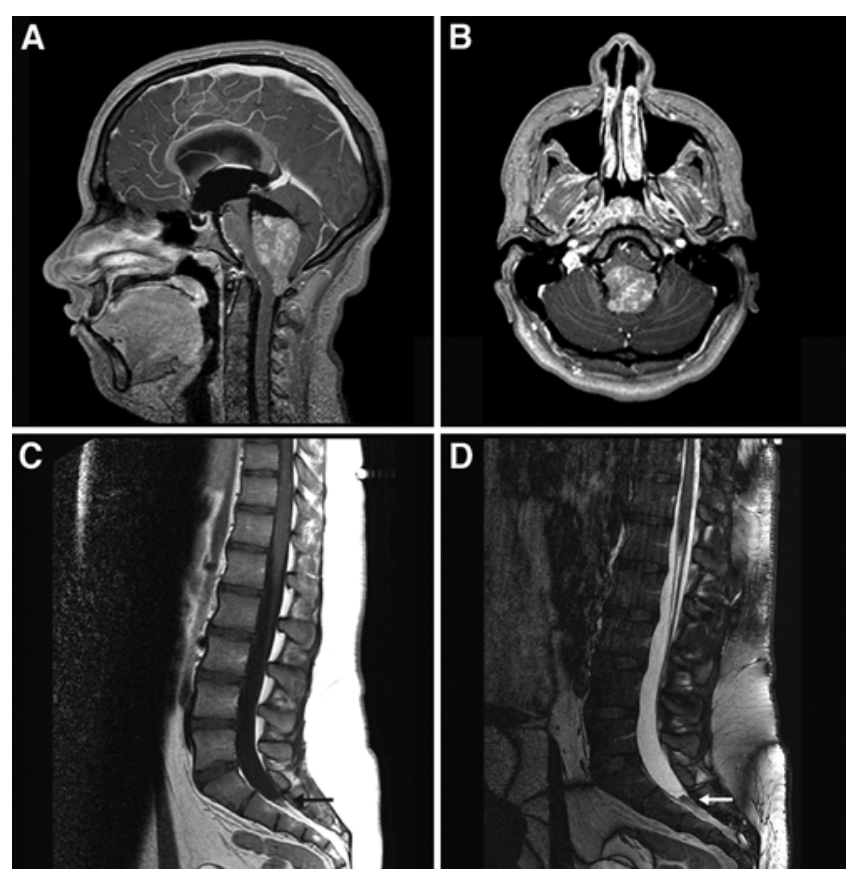

FIG. 1. Preoperative MR images. A and B: Sagittal (A) and axial (B) postcontrast T1-weighted images showing a $4 \times 3-\mathrm{cm}$ heterogeneously enhancing extraaxial fourth ventricular mass. This mass was associated with significant obstructive hydrocephalus. C and D: Sagittal T1- (C) and T2- (D) weighted images demonstrating a sacral lesion at the bottom of the thecal sac (arrows).

the patient demonstrated mild bilateral upper-extremity dysmetria but otherwise had normal findings on formal neurological testing. Brain MRI demonstrated a $4 \times 3-\mathrm{cm}$ heterogeneously enhancing extraaxial fourth ventricular mass causing local mass effect and obstructive hydrocephalus (Fig. 1A and B). Given initial concern for a malignancy, MRI of the entire spine was also performed and revealed a lesion intertwined within the sacral nerve roots (Fig. 1C and D). The patient was asymptomatic from this sacral lesion.

\section{Operative Course and Pathological Findings}

The patient was subsequently brought to the operating

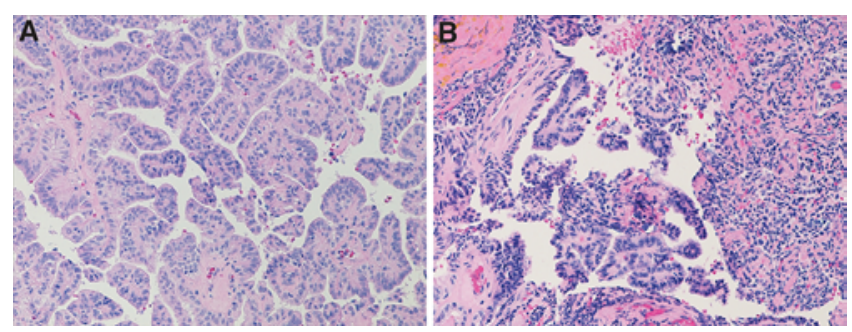

FIG. 2. Photomicrographs. A: Histological examination of the fourth ventricular mass demonstrating classic findings of CPP, including columnar-appearing epithelium surrounding fibrovascular projections. There were fewer than 2 mitoses per $10 \mathrm{hpf}$ on section analysis, and this lesion was classified as a benign CPP (WHO Grade I). B: Similar findings were observed within histological sections of the sacral mass with a diagnosis of benign CPP (WHO Grade I). $\mathrm{H} \& \mathrm{E}$, original magnification $\times 200$. Figure is available in color online only.
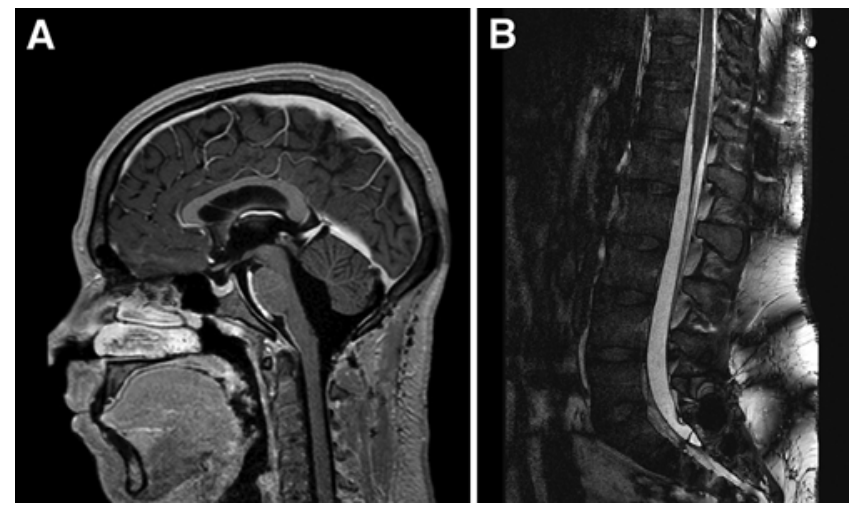

FIG. 3. Postoperative sagittal MR images showing complete resection of both the fourth ventricular (A) and sacral (B) tumor components.

room for a suboccipital craniotomy for tumor resection. Intraoperatively, it was noted that the tumor was moderately tough in consistency and slightly adherent to the floor of the fourth ventricle. A week after the craniotomy, the patient was brought back to the operating room for a sacral laminotomy and resection of the intradural sacral mass. During this procedure, intraoperative ultrasonography was used to confirm the location of the lesion prior to opening the dura mater. A purple-reddish tumor was found at the bottom of the thecal sac intertwined with the sacral nerve roots. Using sharp microdissection and nerve stimulation, the tumor was separated from the nerve roots; postresection neuromonitoring demonstrated no signal changes. Histological examination of both tumors revealed benign CPP (WHO Grade I), with no concerns for a malignant phenotype (Fig. 2).

\section{Postoperative Course}

Postoperative MRI of the brain and spine revealed gross-total resection of both lesions (Fig. 3). The patient's dysmetria improved, and he was weaned off his external ventricular drain and did not require permanent CSF diversion. The patient was eventually discharged home in good condition. Follow-up MRI of both the brain and total spine at 3,6, and 9 months continued to demonstrate no recurrence or new lesions. Given the metastatic presentation of the lesion and young age of the patient, we continue to perform MRI of the brain and total spine every 3 months with a plan to lengthen the imaging interval if scanning findings remain negative.

\section{Discussion}

Choroid plexus tumors are typically categorized histologically according to the WHO grading system: benign CPP (WHO Grade I), atypical CPP (WHO Grade II), and choroid plexus carcinoma (CPC; WHO Grade III). While metastases occur more frequently with CPCs, metastases in benign lesions are thought to be rare. Yet, in adult patients, there are several examples demonstrating that benign CPP can form distant metastases. ${ }^{1-6,8-10,12-14,16-19,22-27,31-36,38,39}$ A review of the literature demonstrated 35 cases in adults (age range 19-74 years, mean 40.3 years; 14 males, 19 females, and 2 patients in whom sex and age were not reported) (Table 1). These included cases in which the metastatic 
TABLE 1. Cases of metastatic CPP in adult patients

\begin{tabular}{|c|c|c|c|c|c|c|c|c|}
\hline $\begin{array}{l}\text { Authors \& } \\
\quad \text { Year }\end{array}$ & $\begin{array}{l}\text { Age } \\
\left(\mathrm{yrs}^{*}\right.\end{array}$ & $\begin{array}{l}\text { Primary } \\
\text { Pathology }\end{array}$ & $\begin{array}{l}\text { Primary } \\
\text { Location }\end{array}$ & $\begin{array}{l}\text { Metastasis } \\
\text { Pathology }\end{array}$ & $\begin{array}{l}\text { Metastasis } \\
\text { Location }\end{array}$ & Metastasis Treatment & $\begin{array}{l}\text { Time to } \\
\text { Metastatic Lesion }\end{array}$ & Outcome \\
\hline $\begin{array}{l}\text { Wilkins \& } \\
\text { Rut- } \\
\text { ledge, } \\
1961\end{array}$ & $56, M$ & CPP & $\begin{array}{l}\text { 4th ven- } \\
\text { tricle }\end{array}$ & $\begin{array}{l}\text { Not avail- } \\
\text { able }\end{array}$ & $\begin{array}{l}\text { Multiple nodules } \\
\text { over cerebellum }\end{array}$ & None & $5 \mathrm{yrs}$ & $\begin{array}{l}\text { Death } 7 \text { yrs after } \\
\text { 1st op }\end{array}$ \\
\hline $\begin{array}{l}\text { Leys et al., } \\
1986\end{array}$ & $40, M$ & CPP & $\begin{array}{l}\text { 4th ven- } \\
\text { tricle }\end{array}$ & CPP & $\begin{array}{l}\text { Local recurrence \& } \\
\text { lat ventricle }\end{array}$ & Not available & 9 yrs & Not available \\
\hline $\begin{array}{l}\text { Girardot } \\
\text { et al., } \\
1990\end{array}$ & $30, F$ & CPP & $\begin{array}{l}\text { Cisterna } \\
\text { magna }\end{array}$ & $\begin{array}{l}\text { Not avail- } \\
\text { able }\end{array}$ & $\begin{array}{l}\text { Local recurrence \& } \\
\text { 4th ventricle }\end{array}$ & Not available & $5 \mathrm{yrs}$ & Not available \\
\hline $\begin{array}{l}\text { Enomoto } \\
\text { et al., } \\
1991\end{array}$ & $46, F$ & CPP & Lt CPA & CPP & Rt temporal region & $\begin{array}{l}\text { Staged resection of It } \\
\text { CPA \& rt temporal } \\
\text { lesions }\end{array}$ & $\begin{array}{l}\text { Present on initial } \\
\text { imaging }\end{array}$ & $\begin{array}{l}\text { Mild deafness imme- } \\
\text { diately postop }\end{array}$ \\
\hline $\begin{array}{l}\text { Domingues } \\
\text { et al., } \\
1991\end{array}$ & $35, F$ & CPP & $\begin{array}{l}\text { Lt lat } \\
\text { foramen } \\
\text { magnum }\end{array}$ & $\begin{array}{l}\text { Not avail- } \\
\text { able }\end{array}$ & $\begin{array}{l}\text { Multiple lesions at } \\
\text { L3-4 }\end{array}$ & $\begin{array}{l}\text { Lumbar lesions not } \\
\text { treated }\end{array}$ & $\begin{array}{l}\text { Present on initial } \\
\text { imaging }\end{array}$ & Not stated \\
\hline $\begin{array}{l}\text { Niikawa et } \\
\text { al., } 1993\end{array}$ & $38, M$ & CPP & $\begin{array}{l}\text { 4th ven- } \\
\text { tricle }\end{array}$ & $\begin{array}{l}\text { CPP (de- } \\
\text { scription } \\
\text { notes } \\
\text { atypical } \\
\text { cells) }\end{array}$ & $\begin{array}{l}\text { Lt CPA \& suprasel- } \\
\text { lar region; } \\
\text { lumbar spinal } \\
\text { lesions found } \\
\text { later }\end{array}$ & $\begin{array}{l}\text { Staged resection of } \\
\text { suprasellar \& It CPA } \\
\text { lesion; whole-brain } \\
\text { RT \& RT to lumbar } \\
\text { spinal lesions }\end{array}$ & $6 \mathrm{yrs}$ & $\begin{array}{l}\text { Mild limb ataxia after } \\
\text { last op w/ stable } \\
\text { disease } 8 \text { mos } \\
\text { after }\end{array}$ \\
\hline $\begin{array}{l}\text { Shake- } \\
\text { speare } \\
\text { et al., } \\
1997\end{array}$ & $27, \mathrm{~F}$ & $\begin{array}{r}\text { Benign } \\
\text { CPP }\end{array}$ & $\begin{array}{l}\text { 4th ven- } \\
\text { tricle }\end{array}$ & Benign CPP & $\begin{array}{l}2 \text { local recurrences } \\
\text { w/ additional } \\
\text { metastases at } \\
\text { It trigeminal } \\
\text { ganglia, along rt } \\
\text { acoustic nerve, } \\
\text { It cerebellum, \& } \\
\text { along spinal axis }\end{array}$ & $\begin{array}{l}\text { Re-resection of 4th } \\
\text { ventricular tumor; } \\
\text { vincristine, carbopla- } \\
\text { tin, \& cyclophospha- } \\
\text { mide but new nodule } \\
\text { found after; followed } \\
\text { by craniospinal RT }\end{array}$ & $\begin{array}{l}2 \text { yrs for local re- } \\
\text { currence, } 3 \text { yrs } \\
\text { until diffuse } \\
\text { metastases }\end{array}$ & $\begin{array}{l}\text { Patient remained } \\
\text { symptom free } \\
13 \text { mos after } \\
\text { craniospinal RT } \\
\text { w/ disease } \\
\text { regression in } \\
\text { lumbosacral area } \\
\text { \& stable disease } \\
\text { elsewhere }\end{array}$ \\
\hline $\begin{array}{c}\text { Leblanc } \\
\text { et al., } \\
1998\end{array}$ & $19, F$ & $\begin{array}{r}\text { Benign } \\
\text { CPP }\end{array}$ & $\begin{array}{l}\text { 4th ven- } \\
\text { tricle }\end{array}$ & $\begin{array}{l}\text { Leptome- } \\
\text { ningeal } \\
\text { choroid } \\
\text { plexus } \\
\text { cysts }\end{array}$ & $\begin{array}{l}\text { Innumerous } \\
\text { cranial \& spinal } \\
\text { leptomeningeal } \\
\text { lesions }\end{array}$ & $\begin{array}{l}\text { 4th ventricular tumor } \\
\text { removed w/ removal } \\
\text { of a few leptomenin- } \\
\text { geal cysts in passing }\end{array}$ & $\begin{array}{l}\text { Present on initial } \\
\text { imaging }\end{array}$ & $\begin{array}{l}\text { Diplopia postop; } \\
\text { MRI } 3 \text { yrs postop } \\
\text { showed enlarge- } \\
\text { ment of residual } \\
\text { cysts but patient } \\
\text { remained asymp- } \\
\text { tomatic }\end{array}$ \\
\hline $\begin{array}{c}\text { Talacchi } \\
\text { et al., } \\
1999\end{array}$ & $38, M$ & CPP & Rt CPA & $\begin{array}{l}\text { Not avail- } \\
\text { able }\end{array}$ & $\begin{array}{l}\text { Local recurrence } \\
\quad \& \text { suprasellar } \\
\text { metastasis }\end{array}$ & $\begin{array}{l}\text { SRS to suprasellar } \\
\text { \& recurrent CPA } \\
\text { lesions }\end{array}$ & $5 \mathrm{yrs}$ & $\begin{array}{l}\text { Died } 7 \text { yrs after 1st } \\
\text { presentation }\end{array}$ \\
\hline $\begin{array}{l}\text { Valencak } \\
\text { et al., } \\
2000\end{array}$ & $33, F$ & CPP & $\begin{array}{l}\text { 4th ven- } \\
\text { tricle }\end{array}$ & $\begin{array}{l}\text { Not avail- } \\
\text { able }\end{array}$ & $\begin{array}{l}\text { Local recurrence, } \\
\text { 3rd ventricle \& } \\
\text { spinal axis }\end{array}$ & $\begin{array}{l}\text { Resection of local } \\
\text { recurrence followed } \\
\text { by RT; progression } \\
\text { requiring SRS to lo- } \\
\text { cal recurrence; SRS } \\
\text { to 3rd ventricular } \\
\text { lesion; additional RT } \\
\text { to local recurrence; } \\
\text { clinically worsened } \\
\text { so started CCNU }\end{array}$ & $\begin{array}{l}\text { Residual tumor } \\
\text { after resection } \\
\text { that pro- } \\
\text { gressed } 8 \text { mos } \\
\text { after 1st op; } \\
43 \text { mos for } \\
\text { 3rd ventricular } \\
\text { lesion; } 51 \text { mos } \\
\text { for spinal axis } \\
\text { lesions }\end{array}$ & $\begin{array}{l}\text { Gait disturbance but } \\
\text { otherwise doing } \\
\text { well } 42 \text { mos after } \\
\text { initiation of CCNU }\end{array}$ \\
\hline
\end{tabular}

lesions demonstrated progression to atypical $\mathrm{CPP}^{26,33,38}$ or retained benign features similar to those of the original primary lesion. . $2,4,9,16-18,22,23,25,27,31,33,39$ Furthermore, these metastatic lesions were found within the ventricular sys- tem, posterior fossa (including the cerebellopontine angle), or anterior or middle cranial fossa; diffusely involving the leptomeninges; or along the spinal axis.

Among the cases in adults, the majority of patients 
R. A. Morshed et al.

» CONTINUED FROM PAGE 473

TABLE 1. Cases of metastatic CPP in adult patients

\begin{tabular}{|c|c|c|c|c|c|c|c|c|}
\hline $\begin{array}{l}\text { Authors \& } \\
\text { Year }\end{array}$ & $\begin{array}{l}\text { Age } \\
(y r s)^{*}\end{array}$ & $\begin{array}{l}\text { Primary } \\
\text { Pathology }\end{array}$ & $\begin{array}{l}\text { Primary } \\
\text { Location }\end{array}$ & $\begin{array}{l}\text { Metastasis } \\
\text { Pathology }\end{array}$ & $\begin{array}{l}\text { Metastasis } \\
\text { Location }\end{array}$ & Metastasis Treatment & $\begin{array}{c}\text { Time to } \\
\text { Metastatic Lesion }\end{array}$ & Outcome \\
\hline $\begin{array}{l}\text { Irsutti et } \\
\quad \text { al., } 2000\end{array}$ & $48, F$ & $\begin{array}{l}\text { Benign } \\
\text { CPP (no } \\
\text { atypia) }\end{array}$ & $\begin{array}{c}\text { 4th ven- } \\
\text { tricle }\end{array}$ & $\begin{array}{l}\text { Benign } \\
\text { CPP (no } \\
\text { atypia) }\end{array}$ & $\begin{array}{l}\text { Local recurrence } \\
\text { \& suprasellar } \\
\text { region }\end{array}$ & $\begin{array}{l}\text { Resection of local } \\
\text { recurrence; later } \\
\text { resection of supra- } \\
\text { sellar lesion }\end{array}$ & $8 \mathrm{yrs}$ & $\begin{array}{l}\text { Lt CN VI \& VII } \\
\text { palsies \& ataxia; } \\
\text { MRI } 6 \text { mos after } \\
\text { last resection } \\
\text { showed no recur- } \\
\text { rence or distant } \\
\text { seeding }\end{array}$ \\
\hline $\begin{array}{l}\text { Jagielski } \\
\text { et al., } \\
2001\end{array}$ & $50, M$ & $\begin{array}{l}\text { CPP } \\
\qquad \text { (WHO } \\
\text { Grade I) }\end{array}$ & $\begin{array}{l}\text { Either 3rd } \\
\text { or 4th } \\
\text { ventricle }\end{array}$ & $\begin{array}{l}\text { CPP (WHO } \\
\text { Grade } \\
\text { l) for rt } \\
\text { temporal } \\
\text { lesion }\end{array}$ & $\begin{array}{l}\text { Parasellar cisterns; } \\
\text { later It temporal } \\
\text { lesion, rt CPA, } \\
\text { It cerebellar, \& } \\
\text { C1-2 lesions }\end{array}$ & $\begin{array}{l}\text { Resection of 4th } \\
\text { ventricular lesion w/ } \\
\text { postop RT; resection } \\
\text { of rt temporal lesion }\end{array}$ & $\begin{array}{l}\text { Parasellar lesion } \\
\text { on initial imag- } \\
\text { ing; It temporal } \\
\text { lesion, rt CPA, } \\
\text { It cerebel- } \\
\text { lar, \& C1-2 } \\
\text { lesions found } \\
4 \text { yrs after } 1 \text { st } \\
\text { presentation }\end{array}$ & $\begin{array}{l}\text { Died } 5 \text { yrs after } \\
\text { diagnosis }\end{array}$ \\
\hline $\begin{array}{l}\text { Heese et } \\
\quad \text { al., } 2002\end{array}$ & $38, M$ & CPP & $\begin{array}{c}\text { 4th ven- } \\
\text { tricle }\end{array}$ & $\begin{array}{l}\text { Not avail- } \\
\text { able }\end{array}$ & $\begin{array}{l}\text { Leptomeninges } \\
\text { around brain- } \\
\text { stem, interpe- } \\
\text { duncular fossa, } \\
\text { chiasmatic } \\
\text { cistern, \& conus } \\
\text { medullaris }\end{array}$ & $\begin{array}{l}\text { After resection of } \\
\text { 4th ventricular } \\
\text { tumor, spontaneous } \\
\text { regression of all } \\
\text { prior leptomeningeal } \\
\text { areas of enhance- } \\
\text { ment; resection of } \\
\text { local recurrence }\end{array}$ & $\begin{array}{l}\text { Present on initial } \\
\text { imaging; local } \\
\text { recurrence } \\
\text { w/ cyst } 8 \text { mos } \\
\text { after 1st op }\end{array}$ & $\begin{array}{l}\text { Discharged from } \\
\text { 2nd surgery w/ } \\
\text { mild gait distur- } \\
\text { bance \& diplopia }\end{array}$ \\
\hline $\begin{array}{l}\text { McEvoy } \\
\text { et al., } \\
2002\end{array}$ & $51, \mathrm{M}$ & $\begin{array}{l}\text { CPP (no } \\
\text { evi- } \\
\text { dence } \\
\text { of } \\
\text { atypia) }\end{array}$ & $\begin{array}{l}\text { 4th ven- } \\
\text { tricle }\end{array}$ & $\begin{array}{l}\text { CPP (no } \\
\text { evidence } \\
\text { of atypia) }\end{array}$ & $\begin{array}{l}\text { Diffuse craniospinal } \\
\text { metastases in- } \\
\text { cluding foramen } \\
\text { magnum, supra- } \\
\text { sellar, CPA, \& } \\
\text { leptomeningeal } \\
\text { spread from } \\
\text { cervical to lum- } \\
\text { bosacral region }\end{array}$ & $\begin{array}{l}\text { Resection of sacral } \\
\text { lesion }\end{array}$ & $5 \mathrm{yrs}$ & Not stated \\
\hline $\begin{array}{c}\text { Yu et al., } \\
2006\end{array}$ & $30, \mathrm{M}$ & CPP & $\begin{array}{l}\text { 4th ven- } \\
\text { tricle }\end{array}$ & $\begin{array}{l}\text { Atypical } \\
\text { CPP }\end{array}$ & $\begin{array}{l}\text { Spinal lesions btwn } \\
\text { conus medullaris } \\
\text { \& terminal filum, } \\
\text { C3-4 \& T7 }\end{array}$ & $\begin{array}{l}\text { Staged resection of all } \\
3 \text { spinal lesions }\end{array}$ & 19 yrs & $\begin{array}{l}\text { Pain resolved but } \\
\text { experienced } \\
\text { urinary retention, } \\
\text { mild lower-ex- } \\
\text { tremity weak- } \\
\text { ness, \& saddle } \\
\text { anesthesia } 2 \text { mos } \\
\text { after op }\end{array}$ \\
\hline $\begin{array}{l}\text { McCall et } \\
\text { al., } 2006\end{array}$ & $38, F$ & CPP & $\begin{array}{l}\text { Posterior } \\
\text { fossa }\end{array}$ & $\begin{array}{l}\text { CPP (cystic } \\
\text { lesions) }\end{array}$ & $\begin{array}{l}\text { Multiple cystic } \\
\text { lesions involving } \\
\text { frontal lobe, } \\
\text { middle cranial } \\
\text { fossa, cerebel- } \\
\text { lum, brainstem, } \\
\text { \& cervical cord; } \\
\text { small mass in } \\
\text { 4th ventricle }\end{array}$ & TMZ & $8 \mathrm{yrs}$ & $\begin{array}{l}\text { Stable disease at } \\
\sim 17 \text { mos after } \\
\text { initiation of TMZ }\end{array}$ \\
\hline
\end{tabular}


» CONTINUED FROM PAGE 474

TABLE 1. Cases of metastatic CPP in adult patients

\begin{tabular}{|c|c|c|c|c|c|c|c|c|}
\hline $\begin{array}{c}\text { Authors \& } \\
\text { Year }\end{array}$ & $\begin{array}{l}\text { Age } \\
(y r s)^{*}\end{array}$ & $\begin{array}{l}\text { Primary } \\
\text { Pathology }\end{array}$ & $\begin{array}{l}\text { Primary } \\
\text { Location }\end{array}$ & $\begin{array}{l}\text { Metastasis } \\
\text { Pathology }\end{array}$ & $\begin{array}{l}\text { Metastasis } \\
\text { Location }\end{array}$ & Metastasis Treatment & $\begin{array}{l}\text { Time to } \\
\text { Metastatic Lesion }\end{array}$ & Outcome \\
\hline & $22, \mathrm{~F}$ & CPP & $\begin{array}{l}\text { 4th ven- } \\
\text { tricle/lt } \\
\text { cerebel- } \\
\text { lar hemi- } \\
\text { sphere }\end{array}$ & $\begin{array}{l}\text { Not avail- } \\
\text { able }\end{array}$ & $\begin{array}{l}\text { Local recurrence, } \\
\text { suprasellar re- } \\
\text { gion, spinal drop } \\
\text { metastasis }\end{array}$ & $\begin{array}{l}\text { Focal RT to local } \\
\text { recurrence; focal } \\
\text { RT to thoracic to } \\
\text { sacral spine; SRS to } \\
\text { suprasellar region; } \\
\text { TMZ given after } \\
\text { worsening clinical } \\
\text { symptoms; intrathe- } \\
\text { cal MTX after lepto- } \\
\text { meningeal spread } \\
\text { but stopped due to } \\
\text { bacterial meningitis; } \\
\text { switched to cisplatin, } \\
\text { cyclophosphamide, } \\
\text { \& etoposide }\end{array}$ & $\begin{array}{l}2.5 \text { yrs until local } \\
\text { recurrence; } 5 \\
\text { yrs: metasta- } \\
\text { ses to supra- } \\
\text { sellar region \& } \\
\text { spinal column; } \\
9 \text { yrs: diffuse } \\
\text { leptomenin- } \\
\text { geal spread \& } \\
\text { enlargement } \\
\text { of spinal drop } \\
\text { metastases }\end{array}$ & $\begin{array}{l}\text { Continued to be } \\
\text { followed in clinic } \\
\text { after multiagent } \\
\text { chemo w/ most } \\
\text { recent KPS score } \\
60 \%\end{array}$ \\
\hline $\begin{array}{l}\text { Jinhu et al., } \\
2007\end{array}$ & $32, F$ & $\begin{array}{r}\text { Benign } \\
\text { CPP }\end{array}$ & $\begin{array}{c}\text { 4th ven- } \\
\text { tricle }\end{array}$ & $\begin{array}{c}\text { Benign CPP } \\
\text { (thoracic } \\
\text { lesion) }\end{array}$ & $\begin{array}{l}\text { Leptomeningeal } \\
\text { infiltration at } \\
\text { cervicomedullary } \\
\text { junction, T8-9 } \\
\text { lesion }\end{array}$ & $\begin{array}{l}\text { Resection of thoracic } \\
\text { lesion }\end{array}$ & $1 \mathrm{mo}$ & $\begin{array}{l}\text { Spontaneous } \\
\text { regression of } \\
\text { leptomeningeal } \\
\text { enhancement } \\
\text { encasing brain- } \\
\text { stem } 2 \text { mos after } \\
\text { resection }\end{array}$ \\
\hline $\begin{array}{l}\text { Ahn \& Cho, } \\
\quad 2007\end{array}$ & $36, F$ & $\begin{array}{l}\text { Benign } \\
\text { CPP (no } \\
\text { atypism } \\
\text { noted) }\end{array}$ & $\begin{array}{l}\text { 4th ven- } \\
\text { tricle }\end{array}$ & $\begin{array}{l}\text { Benign } \\
\text { CPP (no } \\
\text { atypism } \\
\text { noted) } \\
\text { for both } \\
\text { lesions }\end{array}$ & $\begin{array}{l}\text { Local recurrence of } \\
\text { intracranial tu- } \\
\text { mor, rt cerebellar } \\
\text { lesion, several } \\
\text { lesions btwn L-3 } \\
\text { \& S-1 w/ largest } \\
\text { at S-1 }\end{array}$ & $\begin{array}{l}\text { Staged resection of } \\
\text { cerebellar local } \\
\text { recurrence \& sacral } \\
\text { lesion; adjuvant } \\
\text { CCNU after }\end{array}$ & $6 \mathrm{yrs}$ & Not stated \\
\hline $\begin{array}{l}\text { Ortega- } \\
\text { Martínez } \\
\text { et al., } \\
2007\end{array}$ & $20, \mathrm{~F}$ & CPP & $\begin{array}{l}\text { 4th ven- } \\
\text { tricle }\end{array}$ & CPP & $\begin{array}{l}\text { Lesions in cer- } \\
\text { ebellar tonsils } \\
\text { \& obex, both } \\
\text { cavernous } \\
\text { sinuses, \& spinal } \\
\text { region w/ lesions } \\
\text { by cervical cord } \\
\text { down to sacral } \\
\text { region }\end{array}$ & $\begin{array}{l}\text { Intrathecal Ara-C w/ } \\
\text { systemic cisplatin, } \\
\text { bleomycin, \& vin- } \\
\text { cristine }\end{array}$ & $6 \mathrm{yrs}$ & $\begin{array}{c}\text { Died } \sim 6 \text { yrs after } 1 \mathrm{st} \\
\text { presentation ( } 2 \\
\text { mos after biopsy } \\
\text { of cervical lesion) }\end{array}$ \\
\hline $\begin{array}{l}\text { Kaptanoglu } \\
\text { et al., } \\
2007\end{array}$ & $51, \mathrm{~F}$ & CPP & $\begin{array}{l}\text { 4th ven- } \\
\text { tricle }\end{array}$ & CPP & L4-5 lesion & Lumbar lesion resected & $7 \mathrm{yrs}$ & $\begin{array}{l}\text { At } 1 \mathrm{yr} \text {, neurologi- } \\
\text { cally intact \& pain } \\
\text { free }\end{array}$ \\
\hline $\begin{array}{l}\text { Akil et al., } \\
2008\end{array}$ & $74, \mathrm{M}$ & $\begin{array}{l}\text { CPP } \\
\qquad \text { (WHO } \\
\text { Grade I) }\end{array}$ & $\begin{array}{l}\text { 4th ven- } \\
\text { tricle }\end{array}$ & $\begin{array}{l}\text { Not avail- } \\
\text { able }\end{array}$ & $\begin{array}{l}\text { Multiple thoracic } \\
\text { \& lumbar spine } \\
\text { lesions }\end{array}$ & $\begin{array}{l}\text { Only 4th ventricular } \\
\text { lesion resected }\end{array}$ & $\begin{array}{l}\text { Present on initial } \\
\text { imaging }\end{array}$ & $\begin{array}{l}\text { Patient died } 2 \text { wks } \\
\text { postop due to } \\
\text { aspiration pneu- } \\
\text { monia }\end{array}$ \\
\hline $\begin{array}{l}\text { Palmer et } \\
\text { al., } 2010\end{array}$ & $74, \mathrm{M}$ & $\begin{array}{r}\text { Benign } \\
\text { CPP }\end{array}$ & $\begin{array}{l}\text { 4th ven- } \\
\text { tricle }\end{array}$ & $\begin{array}{l}\text { Atypical } \\
\text { CPP }\end{array}$ & $\begin{array}{l}\text { Local recurrence; } \\
\text { T-5 intradural } \\
\text { extramedullary } \\
\text { lesion }\end{array}$ & $\begin{array}{l}\text { Resection \& RT for } \\
\text { local recurrence; } \\
\text { resection \& RT for } \\
\text { T-5 lesion }\end{array}$ & $\begin{array}{l}13 \text { yrs (local } \\
\text { recurrence), } \\
19 \text { yrs (T-5 } \\
\text { lesion) }\end{array}$ & $\begin{array}{l}\text { Remained } \\
\text { paraplegic after } \\
\text { treatment }\end{array}$ \\
\hline $\begin{array}{l}\text { Al-Abdul- } \\
\text { lah et } \\
\text { al., } 2011\end{array}$ & $35, M$ & CPP & $\begin{array}{l}\text { 4th ven- } \\
\text { tricle }\end{array}$ & $\begin{array}{l}\text { CPP (WHO } \\
\text { Grade I; } \\
\text { subfron- } \\
\text { tal mass) }\end{array}$ & $\begin{array}{l}\text { Both CPAs, sub- } \\
\text { frontal region, } \\
\text { multiple middle \& } \\
\text { posterior cranial } \\
\text { fossa lesions }\end{array}$ & $\begin{array}{l}\text { Resection of subfrontal } \\
\text { mass }\end{array}$ & $16 \mathrm{yrs}$ & Not stated \\
\hline
\end{tabular}


R. A. Morshed et al.

» CONTINUED FROM PAGE 475

TABLE 1. Cases of metastatic CPP in adult patients

\begin{tabular}{|c|c|c|c|c|c|c|c|c|}
\hline $\begin{array}{c}\text { Authors \& } \\
\text { Year }\end{array}$ & $\begin{array}{l}\text { Age } \\
(y r s)^{*}\end{array}$ & $\begin{array}{l}\text { Primary } \\
\text { Pathology }\end{array}$ & $\begin{array}{l}\text { Primary } \\
\text { Location }\end{array}$ & $\begin{array}{l}\text { Metastasis } \\
\text { Pathology }\end{array}$ & $\begin{array}{l}\text { Metastasis } \\
\text { Location }\end{array}$ & Metastasis Treatment & $\begin{array}{c}\text { Time to } \\
\text { Metastatic Lesion }\end{array}$ & Outcome \\
\hline \multirow[t]{2}{*}{$\begin{array}{l}\text { Peyre et } \\
\text { al., } 2012\end{array}$} & $39, F$ & $\begin{array}{r}\text { Benign } \\
\text { CPP }\end{array}$ & $\begin{array}{l}\text { 4th ven- } \\
\text { tricle }\end{array}$ & CPP & $\begin{array}{l}\text { Prepontine cistern } \\
\text { to Meckel's cave }\end{array}$ & $\begin{array}{l}\text { SRS to Meckel's cave } \\
\text { lesion; resection of } \\
\text { 4th ventricular mass; } \\
\text { later resection of } \\
\text { prepontine mass } \\
\text { due to progression }\end{array}$ & $\begin{array}{l}\text { Present on initial } \\
\text { imaging }\end{array}$ & $\begin{array}{l}1 \text { yr after resection } \\
\text { showed small } \\
\text { residual tumor in } \\
\text { Meckel's cave w/ } \\
\text { stable diplopia } \\
\text { after op }\end{array}$ \\
\hline & $46, F$ & Not stated & $\begin{array}{l}\text { 4th ven- } \\
\text { tricle }\end{array}$ & $\begin{array}{l}\text { Benign CPP } \\
\quad \text { (It CPA } \\
\text { lesion, rt } \\
\text { fronto- } \\
\text { temporal } \\
\text { lesion) }\end{array}$ & $\begin{array}{l}\text { Lt CPA, rt cavern- } \\
\text { ous sinus, } \\
\text { temporal lesion }\end{array}$ & $\begin{array}{l}\text { Staged resection of It } \\
\text { CPA \& rt fronto- } \\
\text { temporal lesions; } \\
\text { adjuvant RT after } \\
\text { 2nd op }\end{array}$ & $\begin{array}{l}\text { Present on initial } \\
\text { imaging }\end{array}$ & $\begin{array}{l}5 \text { yrs after initial pre- } \\
\text { sentation lesions } \\
\text { stable in size, } \\
\text { patient neurologi- } \\
\text { cally stable }\end{array}$ \\
\hline $\begin{array}{l}\text { Anderson } \\
\text { et al., } \\
2013\end{array}$ & $58, M$ & CPP & $\begin{array}{l}\text { 4th ven- } \\
\text { tricle }\end{array}$ & Not stated & Lumbosacral spine & $\begin{array}{l}\text { EBRT to lumbar spine; } \\
\text { after progression, } \\
\text { received CCNU, } \\
\text { 6-thioguanine, \& } \\
\text { bevacizumab }\end{array}$ & $\begin{array}{l}5 \text { mos until } \\
\text { lesions w/ } \\
\text { in lumbosa- } \\
\text { cral region; } \\
\text { disease } \\
\text { progression } 3 \\
\text { yrs after EBRT } \\
\text { to spine }\end{array}$ & $\begin{array}{l}\text { Radiographic stabil- } \\
\text { ity of lesions w/ } \\
\text { improved pain } \\
\text { control } 12 \text { mos } \\
\text { following bevaciz- } \\
\text { umab initiation }\end{array}$ \\
\hline $\begin{array}{r}\text { Zachary et } \\
\text { al., } 2014\end{array}$ & $21, \mathrm{~F}$ & CPP & $\begin{array}{l}\text { Involving } \\
\text { 4th ven- } \\
\text { tricle, } \\
\text { brain- } \\
\text { stem, \& } \\
\text { rt PICA }\end{array}$ & $\begin{array}{l}\text { CPP (WHO } \\
\text { Grade I) }\end{array}$ & $\begin{array}{l}\text { Local recurrence } \\
\text { in addition to } \\
\text { lesion in rt } \\
\text { anterior \& middle } \\
\text { fossa regions \& } \\
\text { multiple lesions } \\
\text { from cervical to } \\
\text { sacral spine }\end{array}$ & $\begin{array}{l}\text { Resection of rt } \\
\text { temporal lesion \& } \\
\text { craniospinal RT fol- } \\
\text { lowed by boost RT } \\
\text { to cribriform plate, } \\
\text { brainstem, cervical } \\
\text { \& thoracic spine, \& } \\
\text { cauda equina }\end{array}$ & $8 \mathrm{yrs}$ & $\begin{array}{l}3 \text { yrs after RT, no } \\
\text { recurrence or } \\
\text { progression w/ } \\
\text { patient returning } \\
\text { to work }\end{array}$ \\
\hline $\begin{array}{l}\text { Scholsem } \\
\text { et al., } \\
2012\end{array}$ & $59, \mathrm{~F}$ & $\begin{array}{l}\text { Multifocal } \\
\text { atypical } \\
\text { CPP }\end{array}$ & $\begin{array}{l}\text { Rt lat } \\
\text { ventricle } \\
\text { \& 4th } \\
\text { ventricle } \\
\text { tumors } \\
\text { discov- } \\
\text { ered 1st }\end{array}$ & $\begin{array}{l}\text { Low-grade } \\
\text { CPP (4th } \\
\text { \& rt lat } \\
\text { ventricu- } \\
\text { lar tumor) }\end{array}$ & $\begin{array}{l}\text { 4th ventricle, rt lat } \\
\text { ventricle, It lat } \\
\text { ventricle }\end{array}$ & $\begin{array}{l}\text { Staged resection of 4th } \\
\text { ventricular tumor \& } \\
\text { rt lat ventricular tu- } \\
\text { mor; patient refused } \\
\text { resection of It lat } \\
\text { ventricular tumor }\end{array}$ & $\begin{array}{l}\text { Rt lat \& 4th ven- } \\
\text { tricular tumors } \\
\text { present on } \\
\text { initial scan; It } \\
\text { lat ventricular } \\
\text { discovered } 6 \\
\text { yrs later }\end{array}$ & Not stated \\
\hline $\begin{array}{l}\text { Stuivenvolt } \\
\text { et al., } \\
2012\end{array}$ & $50, \mathrm{~F}$ & $\begin{array}{l}\text { Benign } \\
\text { CPP } \\
\text { (WHO } \\
\text { Grade I) }\end{array}$ & $\begin{array}{l}\text { 4th ven- } \\
\text { tricle }\end{array}$ & $\begin{array}{l}\text { Atypical } \\
\text { CPP } \\
\text { (WHO } \\
\text { Grade II) }\end{array}$ & $\begin{array}{l}\text { Local recurrence, } \\
\text { S1-2 level intra- } \\
\text { dural extramed- } \\
\text { ullary, T-9 level } \\
\text { intradural }\end{array}$ & $\begin{array}{l}\text { Resection of sacral } \\
\text { lesion; RT to cranio- } \\
\text { spinal axis w/ boosts } \\
\text { to relapsed 4th ven- } \\
\text { tricular tumor, T-9, \& } \\
\text { S1-2 regions }\end{array}$ & 6 yrs & $\begin{array}{l}8 \text { yrs after initial } \\
\text { presentation, } \\
\text { no neurologi- } \\
\text { cal complaints; } \\
\text { MRI showed no } \\
\text { change of recur- } \\
\text { rent tumor in 4th } \\
\text { ventricle or at T-9 }\end{array}$ \\
\hline \multirow[t]{2}{*}{$\begin{array}{l}\text { Bettegow- } \\
\text { da et al., } \\
2012\end{array}$} & $\begin{array}{l}\text { Not } \\
\text { stat- } \\
\text { ed }\end{array}$ & CPP & $\begin{array}{l}\text { 4th ven- } \\
\text { tricle }\end{array}$ & Not stated & Lumbosacral spine & Not stated & Not stated & Not stated \\
\hline & $\begin{array}{l}\text { Not } \\
\text { stat- } \\
\text { ed }\end{array}$ & CPP & $\begin{array}{l}\text { 4th ven- } \\
\text { tricle }\end{array}$ & Not stated & Lumbosacral spine & Not stated & Not stated & Not stated \\
\hline
\end{tabular}

had a primary lesion within the fourth ventricle, although this was not essential to metastasis formation. In addition, while some metastatic lesions were discovered in concur- rence with a primary lesion at initial presentation, other groups have reported that evidence of metastasis did not become apparent until years later. Yu et al. reported an 
» CONTINUED FROM PAGE 476

TABLE 1. Cases of metastatic CPP in adult patients

\begin{tabular}{|c|c|c|c|c|c|c|c|c|}
\hline $\begin{array}{c}\text { Authors \& } \\
\text { Year }\end{array}$ & $\begin{array}{l}\text { Age } \\
(\mathrm{yrs})^{*}\end{array}$ & $\begin{array}{l}\text { Primary } \\
\text { Pathology }\end{array}$ & $\begin{array}{l}\text { Primary } \\
\text { Location }\end{array}$ & $\begin{array}{l}\text { Metastasis } \\
\text { Pathology }\end{array}$ & $\begin{array}{l}\text { Metastasis } \\
\text { Location }\end{array}$ & Metastasis Treatment & $\begin{array}{c}\text { Time to } \\
\text { Metastatic Lesion }\end{array}$ & Outcome \\
\hline \multirow[t]{3}{*}{$\begin{array}{l}\text { Abdulkader } \\
\text { et al., } \\
2016\end{array}$} & $19, \mathrm{~F}$ & CPP & $\begin{array}{l}\text { Rt lat } \\
\text { ventricle } \\
\text { (report- } \\
\text { ed to be } \\
\text { multifo- } \\
\text { cal } \\
\text { on 1st } \\
\text { presen- } \\
\text { tation) }\end{array}$ & CPP & $\begin{array}{l}\text { Both lat \& 4th } \\
\text { ventricles; } 3 \text { rd } \\
\text { ventricular/ } \\
\text { suprasellar } \\
\text { region, rt porus } \\
\text { acusticus, It CN } \\
\text { VI, posterior } \\
\text { cerebellar vermis }\end{array}$ & $\begin{array}{l}\text { Resection of } 3 \text { rd } \\
\text { ventricular/suprasel- } \\
\text { lar lesion }\end{array}$ & $19 \mathrm{yrs}$ & $\begin{array}{l}\text { Postop developed } \\
\text { diabetes insipi- } \\
\text { dus; follow-up MR } \\
\text { images revealed } \\
\text { slow continuous } \\
\text { growth of mass in } \\
\text { the rt lat ventricle } \\
\text { w/ stable disease } \\
\text { elsewhere }\end{array}$ \\
\hline & $50, M$ & CPP & $\begin{array}{l}\text { 4th ven- } \\
\text { tricle }\end{array}$ & CPP & $\begin{array}{l}\text { Multiple posterior } \\
\text { fossa lesions \& } \\
\text { spinal leptome- } \\
\text { ningeal lesions }\end{array}$ & $\begin{array}{l}\text { Resection of 4th } \\
\text { ventricular \& large } \\
\text { CPA lesions; radio- } \\
\text { surgery required } \\
\text { starting } 12 \text { yrs later; } \\
\text { eventually TMZ }\end{array}$ & $\begin{array}{l}\text { Present on initial } \\
\text { imaging }\end{array}$ & $\begin{array}{l}\text { TMZ did not lead to } \\
\text { tumor regression } \\
\text { \& patient died }\end{array}$ \\
\hline & $27, \mathrm{M}$ & CPP & $\begin{array}{l}\text { 4th ven- } \\
\text { tricle }\end{array}$ & CPP & $\begin{array}{l}\text { 3rd ventricle, mul- } \\
\text { tiple 4th ventricle } \\
\text { lesions, pineal } \\
\text { region }\end{array}$ & $\begin{array}{l}\text { Resection \& radiosur- } \\
\text { gery for local recur- } \\
\text { rence; radiosurgery } \\
\text { for distant spread }\end{array}$ & 25 yrs & $\begin{array}{l}\text { Died of unknown } \\
\text { causes }\end{array}$ \\
\hline
\end{tabular}

$\mathrm{CCNU}=$ lomustine; chemo = chemotherapy; $\mathrm{CPA}=$ cerebellopontine angle; $\mathrm{CN}=$ cranial nerve; $\mathrm{EBRT}=$ external-beam RT; $\mathrm{KPS}=$ Karnofsky Performance Scale; $\mathrm{MTX}=$ methotrexate; $\mathrm{PICA}=$ posterior inferior cerebellar artery; $\mathrm{RT}$ = radiotherapy; $\mathrm{SRS}$ = stereotactic radiosurgery; $\mathrm{TMZ}=$ temozolomide.

* Age at the first presentation.

extreme example in which a patient developed diffuse spinal lesions 19 years after his initial diagnosis and resection. ${ }^{38}$ It is unclear whether these differences in time to metastasis are due to seeding at the time of surgery or by innate tumor cell spread within the CSF. Based on the literature and prior reports, it is likely that tumor cells originating within the fourth ventricle have more direct access to the subarachnoid space outside the ventricular pathway through the foramen of Magendie and foramina of Luschka. This may also account for the fewer number of reports of metastatic CPPs in pediatric patients, in whom supratentorial locations are more common. ${ }^{15,37}$

In pediatric patients, in contrast to adults, there are very few reports of benign metastasis or drop metastasis. A review of the literature demonstrated 3 cases, with 2 males and 1 female ranging from 3 to 16 years of age (mean 8.7 years; Table 2). Doglietto et al. reported on a 16-year-old boy who, on initial presentation, was found to have diffuse solid lesions in the cisterna magna, both cerebellopontine angles, and lumbosacral space. Surgical removal of the solid and cystic lesions in the cisterna magna confirmed a diagnosis of CPP. ${ }^{7}$ Guidetti and Spallone reported on a 3 -year-old girl with a third ventricular CPP that was resected. Unfortunately, 3 years later this patient was found to have a new fourth ventricular mass, which was theorized to be due to tumor seeding rather than an intrinsic lesion originating from the fourth ventricle choroid plexus itself. ${ }^{11}$ However, the pathological diagnosis and histological characteristics of this new mass were never examined. Masuzawa et al. reported on a 7-year-old patient who was diagnosed with a fourth ventricular CPP and eventually underwent resection of the tumor 9 years after the initial diagnosis. Approximately 4 years after the initial operation, the patient developed symptoms of cervical cord

TABLE 2. Cases of metastatic CPP in pediatric patients

\begin{tabular}{|c|c|c|c|c|c|c|c|c|}
\hline Authors \& Year & $\begin{array}{l}\text { Age } \\
(\mathrm{yrs})^{*}\end{array}$ & $\begin{array}{l}\text { Primary } \\
\text { Pathology }\end{array}$ & $\begin{array}{l}\text { Primary } \\
\text { Location }\end{array}$ & $\begin{array}{l}\text { Metastasis } \\
\text { Pathology }\end{array}$ & $\begin{array}{l}\text { Metastasis } \\
\text { Location }\end{array}$ & $\begin{array}{l}\text { Treatment of } \\
\text { Metastasis }\end{array}$ & $\begin{array}{c}\text { Time to } \\
\text { Metastatic Lesion }\end{array}$ & Outcome \\
\hline $\begin{array}{l}\text { Guidetti \& Spal- } \\
\text { lone, } 1981\end{array}$ & $3, \mathrm{~F}$ & CPP & 3rd ventricle & Not available & 4th ventricle & $\begin{array}{l}\text { Family refused } \\
\text { surgery }\end{array}$ & 3 yrs & Not stated \\
\hline $\begin{array}{l}\text { Masuzawa et } \\
\text { al., } 1981\end{array}$ & $7, \mathrm{M}$ & CPP & $\begin{array}{r}\text { 4th ventricle \& } \\
\text { CPA region }\end{array}$ & CPP & $\begin{array}{l}\text { C6-7 cervi- } \\
\text { cal region }\end{array}$ & Subtotal resection & $13 \mathrm{yrs}$ & Not stated \\
\hline $\begin{array}{l}\text { Doglietto et al., } \\
2005\end{array}$ & $16, M$ & CPP & $\begin{array}{l}\text { Cisterna } \\
\text { magna }\end{array}$ & $\begin{array}{c}\text { CPP (cisterna } \\
\text { magna cys- } \\
\text { tic lesions) }\end{array}$ & $\begin{array}{l}\text { Multiple cra- } \\
\text { niospinal } \\
\text { lesions }\end{array}$ & $\begin{array}{l}\text { Resection of cis- } \\
\text { terna magna solid } \\
\text { \& cystic lesions }\end{array}$ & $\begin{array}{l}\text { Present on initial } \\
\text { imaging }\end{array}$ & $\begin{array}{l}\text { Lt hypoacusia } 1 \mathrm{yr} \\
\text { postop w/o signs of } \\
\text { disease progression }\end{array}$ \\
\hline
\end{tabular}

* Age at the first presentation. 
compression with a mass lesion found at the C6-7 region, with histological findings being consistent with a well-differentiated CPP, albeit with more nuclear pleomorphism and mitotic figures. ${ }^{20}$ Although not stated, this metastatic lesion may have been classified as an atypical CPP. Here, we describe a pediatric patient who presented with both a benign (WHO Grade I) CPP within the fourth ventricle and a sacral drop metastasis concurrently, prior to any surgical intervention. This finding, in conjunction with the case reported by Doglietto et al., suggests that, although rare, CPPs have an intrinsic ability to metastasize not only in adult patients but also in children.

With this evidence in mind, the workup and long-term management of patients with benign CPPs may need to be reexamined to incorporate the possibility of metastases. For fourth ventricular tumors in a pediatric patient, most practitioners would obtain an MRI study of the entire spine at initial presentation, given the concern for drop metastases from possible medulloblastoma or ependymoma. In patients who may not have undergone spine imaging on initial workup for various reasons, we believe that immediate postoperative imaging of the spine should be performed. Given this case and other reports, it can be argued that MRI of the entire spinal axis on surveillance imaging is also warranted even in patients with benign CPPs (WHO Grade I). This approach has been advocated previously in the context of adult patients, but less so in the pediatric population. ${ }^{33,38}$ As there were some cases in which patients died of spread of their primary tumor, catching a metastatic lesion at initial presentation or earlier on follow-up imaging may prevent such devastating outcomes.

It is unclear what the best treatment strategies are for patients with disseminated disease and if there are prophylactic measures that may be taken to prevent metastasis. Although radiotherapy, stereotactic radiosurgery, and various chemotherapy agents have been used, in some cases with success, there are no standard treatment algorithms for metastatic CPP lesions, and no report has demonstrated the use of adjuvant therapies in pediatric patients. In some reports, metastatic lesions causing significant neurological deficits were resected, but resection in cases of extensive disseminated lesions was limited. With the understanding that each case is unique and challenging in its own manner, our recommendation for the management of benign CPPs with CSF spread and metastasis is generally to remove all lesions if possible, because this approach can be curative. Given the rarity of these cases, however, a prospective clinical trial or even a retrospective comparative study may not be feasible. Moreover, there are no clear guidelines for the length of follow-up needed for these patients, depending strongly on practice habits. As metastases have been found up to 19 years after initial presentation, it may be especially important in the pediatric patient to be attentive to signs and symptoms of potential slow-growing distant lesions. Again, we advocate imaging of the brain and total spine during follow-up to ensure detection of potential new sites of growth.

\section{Conclusions}

Here, we report the case of a metastatic benign sacral
CPP discovered at the time of initial presentation in a patient with a benign fourth ventricular CPP. Both lesions were treated successfully with resection alone, and followup is currently ongoing. Although metastatic spread of a benign CPP is a rare occurrence in the pediatric population, this case, in conjunction with reports within the literature, illustrates the utility of undertaking imaging of the entire spinal axis at the time of diagnosis. By doing so, metastatic lesions may undergo early intervention, preventing poor outcomes such as disabling neurological deficits or even death.

\section{References}

1. Abdulkader MM, Mansour NH, Van Gompel JJ, Bosh GA, Dropcho EJ, Bonnin JM, et al: Disseminated choroid plexus papillomas in adults: A case series and review of the literature. J Clin Neurosci 32:148-154, 2016

2. Ahn SS, Cho YD: Spinal drop metastasis from a posterior fossa choroid plexus papilloma. J Korean Neurosurg Soc 42:475-477, 2007

3. Akil H, Coupe NJ, Singh J: Spinal deposits of a benign choroid plexus papilloma. J Clin Neurosci 15:708-712, 2008

4. Al-Abdullah AA, Abu-Amero KK, Hellani A, Alkhalidi H, Bosley TM: Choroid plexus papilloma metastases to both cerebellopontine angles mimicking neurofibromatosis type 2 . J Neurol 258:504-506, 2011

5. Anderson MD, Theeler BJ, Penas-Prado M, Groves MD, Yung WK: Bevacizumab use in disseminated choroid plexus papilloma. J Neurooncol 114:251-253, 2013

6. Bettegowda C, Adogwa O, Mehta V, Chaichana KL, Weingart J, Carson BS, et al: Treatment of choroid plexus tumors: a 20-year single institutional experience. J Neurosurg Pediatr 10:398-405, 2012

7. Doglietto F, Lauretti L, Tartaglione T, Gessi M, Fernandez E, Maira G: Diffuse craniospinal choroid plexus papilloma with involvement of both cerebellopontine angles. Neurology 65:842, 2005

8. Domingues RC, Taveras JM, Reimer P, Rosen BR: Foramen magnum choroid plexus papilloma with drop metastases to the lumbar spine. AJNR Am J Neuroradiol 12:564-565, 1991

9. Enomoto H, Mizuno M, Katsumata T, Doi T: Intracranial metastasis of a choroid plexus papilloma originating in the cerebellopontine angle region: a case report. Surg Neurol 36:54-58, 1991

10. Girardot C, Boukobza M, Lamoureux JP, Sichez JP, Capelle L, Zouaoui A, et al: Choroid plexus papillomas of the posterior fossa in adults: MR imaging and gadolinium enhancement. Report of four cases and review of the literature. J Neuroradiol 17:303-318, 1990

11. Guidetti B, Spallone A: The surgical treatment of choroid plexus papillomas: the results of 27 years experience. Neurosurg Rev 4:129-137, 1981

12. Heese O, Lamszus K, Grzyska U, Westphal M: Diffuse arachnoidal enhancement of a well differentiated choroid plexus papilloma. Acta Neurochir (Wien) 144:723-728, 2002

13. Irsutti M, Thorn-Kany M, Arrué P, Richaud J, Sol JC, Delisle $\mathrm{MB}$, et al: Suprasellar seeding of a benign choroid plexus papilloma of the fourth ventricle with local recurrence. Neuroradiology 42:657-661, 2000

14. Jagielski J, Zabek M, Wierzba-Bobrowicz T, Łakomiec B, Chrapusta SJ: Disseminating histologically benign multiple papilloma of the choroid plexus: case report. Folia Neuropathol 39:209-213, 2001

15. Jänisch W, Staneczek W: [Primary tumors of the choroid plexus. Frequency, localization and age.] Zentralbl Allg Pathol 135:235-240, 1989 (Ger) 
16. Jinhu Y, Jianping D, Jun M, Hui S, Yepeng F: Metastasis of a histologically benign choroid plexus papilloma: case report and review of the literature. J Neurooncol 83:47-52, 2007

17. Kaptanoglu E, Tun K, Celikmez RC, Ozen O, Taskin Y: Spinal drop metastasis of choroid plexus papilloma. J Clin Neurosci 14:381-383, 2007

18. Leblanc R, Bekhor S, Melanson D, Carpenter S: Diffuse craniospinal seeding from a benign fourth ventricle choroid plexus papilloma. Case report. J Neurosurg 88:757-760, 1998

19. Leys D, Pasquier F, Lejeune JP, Lesoin F, Petit H, Delandsheer JM: [Benign choroid plexus papilloma. 2 local recurrences and intraventricular seeding.] Neurochirurgie 32:258-261, 1986 (Fr)

20. Masuzawa T, Shimabukuro H, Yoshimizu N, Sato F: Ultrastructure of disseminated choroid plexus papilloma. Acta Neuropathol 54:321-324, 1981

21. Matson DD, Crofton FD: Papilloma of the choroid plexus in childhood. J Neurosurg 17:1002-1027, 1960

22. McCall T, Binning M, Blumenthal DT, Jensen RL: Variations of disseminated choroid plexus papilloma: 2 case reports and a review of the literature. Surg Neurol 66:62-68, 2006

23. McEvoy AW, Galloway M, Revesz T, Kitchen ND: Metastatic choroid plexus papilloma: a case report. J Neurooncol 56:241-246, 2002

24. Niikawa S, Ito T, Murakawa T, Hirayama H, Ando T, Sakai $\mathrm{N}$, et al: Recurrence of choroid plexus papilloma with malignant transformation-case report and lectin histochemistry study. Neurol Med Chir (Tokyo) 33:32-35, 1993

25. Ortega-Martínez M, Cabezudo-Artero JM, FernándezPortales I, Pimentel JJ, Gómez de Tejada R: Diffuse leptomeningeal seeding from benign choroid plexus papilloma. Acta Neurochir (Wien) 149:1229-1237, 2007

26. Palmer C, Kalsi P, Scoones D, Bradey N, Strachan R: Recurrence of fourth ventricular choroid plexus papilloma with late occurrence of a spinal drop metastasis. Br J Neurosurg 24:482-484, 2010

27. Peyre M, Bah A, Kalamarides M: Multifocal choroid plexus papillomas: case report. Acta Neurochir (Wien) 154:295299, 2012

28. Safaee M, Clark AJ, Bloch O, Oh MC, Singh A, Auguste KI, et al: Surgical outcomes in choroid plexus papillomas: an institutional experience. J Neurooncol 113:117-125, 2013

29. Safaee M, Oh MC, Bloch O, Sun MZ, Kaur G, Auguste KI, et al: Choroid plexus papillomas: advances in molecular biology and understanding of tumorigenesis. Neuro Oncol 15:255267,2013

30. Safaee M, Oh MC, Sughrue ME, Delance AR, Bloch O, Sun $\mathrm{M}$, et al: The relative patient benefit of gross total resection in adult choroid plexus papillomas. J Clin Neurosci 20:808 812,2013
31. Scholsem M, Scholtes F, Robe PA, Bianchi E, Kroonen J, Deprez M: Multifocal choroid plexus papilloma: a case report. Clin Neuropathol 31:430-434, 2012

32. Shakespeare TP, Slancar MM, Mallik AR, Bell DR: CSF dissemination of a benign choroid plexus papilloma (CPP). Aust N Z J Med 27:597-598, 1997

33. Stuivenvolt M, Mandl E, Verheul J, Fleischeuer R, Tijssen CC: Atypical transformation in sacral drop metastasis from posterior fossa choroid plexus papilloma. BMJ Case Rep 2012:bcr0120125681, 2012

34. Talacchi A, De Micheli E, Lombardo C, Turazzi S, Bricolo A: Choroid plexus papilloma of the cerebellopontine angle: a twelve patient series. Surg Neurol 51:621-629, 1999

35. Valencak J, Dietrich W, Raderer M, Dieckmann K, Prayer D, Hainfellner JA, et al: Evidence of therapeutic efficacy of $\mathrm{CCNU}$ in recurrent choroid plexus papilloma. $\mathbf{J}$ Neurooncol 49:263-268, 2000

36. Wilkins H, Rutledge BJ: Papillomas of the choroid plexus. J Neurosurg 18:14-18, 1961

37. Wolff JE, Sajedi M, Brant R, Coppes MJ, Egeler RM: Choroid plexus tumours. Br J Cancer 87:1086-1091, 2002

38. Yu H, Yao TL, Spooner J, Stumph JR, Hester R, Konrad PE: Delayed occurrence of multiple spinal drop metastases from a posterior fossa choroid plexus papilloma. Case report. J Neurosurg Spine 4:494-496, 2006

39. Zachary G, George J, Jaishri B, Peter B, Stephanie T: Management of disseminated choroid plexus papilloma: a case study. Pediatr Blood Cancer 61:562-563, 2014

\section{Disclosures}

The authors report no conflict of interest concerning the materials or methods used in this study or the findings specified in this paper.

\section{Author Contributions}

Conception and design: all authors. Acquisition of data: Lau, Sun, Ostling. Analysis and interpretation of data: all authors. Drafting the article: all authors. Critically revising the article: all authors. Reviewed submitted version of manuscript: Lau, Ostling. Approved the final version of the manuscript on behalf of all authors: Morshed.

\section{Correspondence}

Ramin A. Morshed, Department of Neurological Surgery, University of California, San Francisco, 505 Parnassus Ave., Rm. M-779, San Francisco, CA 94143-0112. email: ramin.morshed@ucsf.edu. 